Original Article

\title{
A preliminary study of the measurement of overload applied to the foot support of a wheelchair and a seated postural support device
}

\author{
Satoshi Shirogane, RPT, PhD ${ }^{1)^{*}}$, TAKashi Handa, PhD ${ }^{2)}$, Yoshihiko Kozai, MS $^{2)}$, \\ Yusuke MAEDA, RPT, PhD ${ }^{3}$ \\ 1) Research Institute, National Rehabilitation Center for Persons with Disabilities: 4-1 Namiki, \\ Tokorozawa 359-8555, Japan \\ 2) Saitama Industrial Technology Center, Japan \\ 3) Department of Physical Therapy, Faculty of Health Sciences, Mejiro University, Japan
}

\begin{abstract}
Purpose] The mechanical strength of wheelchair and seating products is specified by standards to ensure user safety. However, some individual users are affected by severe spasticity resulting from cerebral palsy that occasionally breaks such devices. Until recently, reports of quantitative measurement of these mechanical overloads have been scarce, and the exact loads applied have been unknown. This study aimed to conduct a sampling survey prior to conducting a future full-scale study. [Subjects and Methods] Using two force plates, we measured the load on the foot support applied by three participants with cerebral palsy. The applied load was measured at a $100 \mathrm{~Hz}$ sampling rate, and maximum load and vertical/horizontal component forces were analyzed. [Results] The maximum load applied by participants was approximately 1.34 times their body weight. The vertical force applied on the foot support accounted for $70-80 \%$ of the total load. The horizontal force also reached about $35 \%$ at its maximum. [Conclusion] Because the horizontal load also reached $1 / 3$ of the total load, it will be advantageous to use a threeaxis force plate to measure forces in a future study with more participants.

Key words: Wheelchair, Foot support, Mechanical strength
\end{abstract}

(This article was submitted Jun. 19, 2016, and was accepted Sep. 14, 2016)

\section{INTRODUCTION}

Being able to take and maintain a seated posture is extremely important in daily life. Thus, the evaluation and training of seated posture in patients are often performed in physical therapy ${ }^{1)}$. For individuals who are incapable of holding a seated posture position even after physical therapy intervention, assistive devices such as wheelchairs or seated postural support are used. Because these devices are essential for these individuals, in Japan, these devices are covered by a grant for prosthetic device expenses under the Services and Supports for Persons with Disabilities Act.

Wheelchairs and seated postural support devices are routinely used in daily life, so they must be highly reliable and durable. Their mechanical strengths are defined by associated industrial standards, including the International Organization for Standardization (ISO) ${ }^{2}$, Japanese Industrial Standards ${ }^{3)}$, or criteria established by the Ministry of Health, Labour, and Welfare $^{4)}$ to ensure user safety.

Involuntary muscle contraction ${ }^{5)}$ (spasticity) may occur in users with diseases of impaired motor control function, including cerebral palsy and hemiplegia caused by stroke. Spasticity occasionally causes unintended strong muscle contraction and movement, which may result in overload that exceeds the recommended standards for the wheelchair or seated postural support device. Indeed, cases of damage to wheelchairs or seated postural support devices caused by a strong involuntary

*Corresponding author. Satoshi Shirogane (E-mail: shirogane-satoshi@rehab.go.jp)

(C2017 The Society of Physical Therapy Science. Published by IPEC Inc.

This is an open-access article distributed under the terms of the Creative Commons Attribution Non-Commercial No Derivatives (by-nc-nd) License $<$ http://creativecommons.org/licenses/by-nc-nd/4.0/>. 
movement resulting from spasticity have been reported in Japan ${ }^{6,7)}$. As breaking these devices is associated with the risk of a serious accident, such events should be avoided as much as possible. To develop and supply safer devices to users, it is necessary to improve current test methods and guidelines for heavier loads; however, few studies have quantified these overloads, and specific reference values have not been defined yet.

Therefore, we previously conducted a quantitative study of the load on such devices with the following aims: to define the overload value among disabled individuals with strong spasticity; review the test methods and standard values to prevent hazards to users of wheelchairs and seated postural support devices; and determine a safer environment for these users. Among several reported breakage sites, we focused on the foot support, and then we prepared to perform the measurements. The ISO standard for wheelchairs ${ }^{8)}$ stipulates the testing method of the foot support as follows: place a predetermined load vertically on the foot support surface and confirm the burden (Fig. 1). The standard value in this test varies depending on the user's body weight as follows: $250 \mathrm{~N}$ for persons of $25 \mathrm{~kg}$ or less, $500 \mathrm{~N}$ for persons of $50 \mathrm{~kg}$ or less, $750 \mathrm{~N}$ for persons of $75 \mathrm{~kg}$ or less, and $1,000 \mathrm{~N}$ for persons of $100 \mathrm{~kg}$ or less. However, damage to wheelchairs and seated postural support devices that satisfy the aforementioned criteria has been reported; thus, this test method and these standard values may not be sufficient to ensure safety. It is assumed that loads are placed on the foot support from other directions as well as the vertical direction in users observed in clinical practice. If these effects are large, the establishment of other test methods and standard values is required to account for three-dimensional loads and vertical forces.

Intended to be a precursor to a full-scale future study, the present study is a preliminary sampling survey that aims to confirm the measurement method and obtain the reference values for forces on the foot support surface from the vertical and other directions. If a stronger force is observed from other directions than in the vertical direction, even in one case, then the need for a measurement that considers the force from other directions will be assessed in a full-scale study.

\section{SUBJECTS AND METHODS}

This study included individuals with quadriplegia caused by cerebral palsy who mainly used seated postural support devices. Three individuals (a 17-year-old male, a 31-year-old male, and a 48-year-old female) who had severe spastic cerebral palsy participated. Before participating, an explanation about the study methods and risks was provided in writing, and informed consent was obtained from all participants. This study was conducted after obtaining approval from the ethical review committee of the National Rehabilitation Center for Persons with Disabilities (no. 26-138).

Wheelchairs and seated postural support devices that were commonly used by the participants were used to measure the actual overload on devices as much as possible. Seating posture and the use of support devices at the time of the measurement depended on the daily situation of the individual. The loads on the foot support were measured by placing two small, transportable force plates (M3DFP, Tec Gihan Co., Ltd.) between both of the participant's feet and the foot support (Fig. 2). The measurement was repeated one to three times at a sampling rate of $100 \mathrm{~Hz}$, depending on the participant's health condition, and a single measurement took up to ten minutes. Output from the force plates was measured and recorded via a USB cable that was connected to a notebook computer using software. Obtained values were provided in a comma-separated value format for the three axes $(F x, F y$, and $F z)$ of each force plate.

First, for time $t$, the sum of the measured values from each axis of each force plate was defined as the total force on the foot support. Second, the length of the three-dimensional vector, $L_{(t)}$, was calculated as follows:

$$
L_{(t)}=\sqrt{F x_{(t)}^{2}+F y_{(t)}^{2}+F z_{(t)}^{2}}
$$

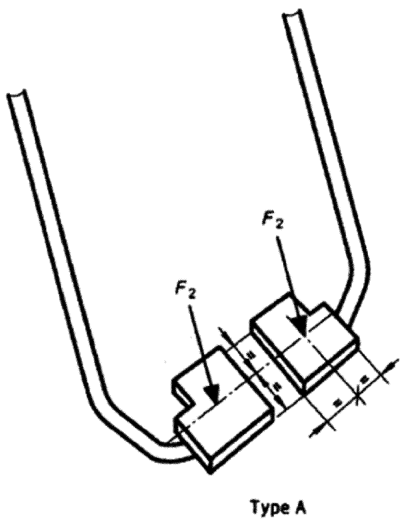

Fig. 1. Foot support test method of the International Organization for Standardization ${ }^{8)}$

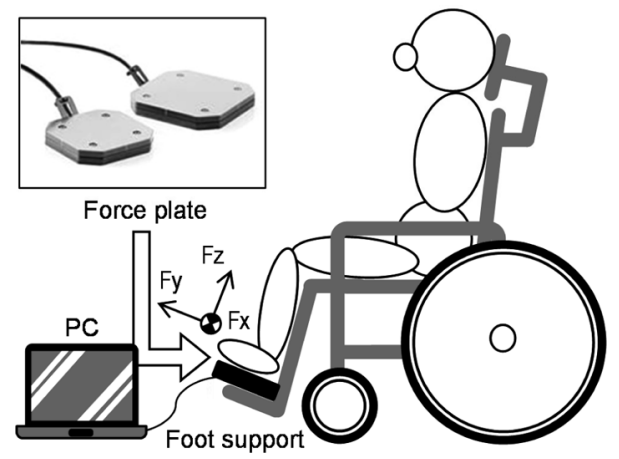

Fig. 2. Experimental setting

PC: personal computer; Fz: vertical force on the foot support; Fx and Fy: horizontal forces on the foot support 
To evaluate the amount of vertical force on the foot support, the time $\left(t_{\max }\right)$ when the maximum load was placed on the foot support was calculated from the obtained $L_{(t)}$, and each 5 -second period before and after $t_{\max }$ (ten seconds in total) was analyzed. Temporal changes were compared between the vertical force on the foot support $(F z)$ and the sum of horizontal forces on the foot support $(F x+F y)$. The ratios of the vertical force and horizontal forces to the sum of the three forces (\%Vertical, \%Horizontal) were calculated as follows:

$$
\begin{aligned}
& \% \text { Vertical }=\frac{F z}{F x+F y+F z} \times 100 \\
& \% \text { Horizontal }=\frac{F x+F y}{F x+F y+F z} \times 100
\end{aligned}
$$

In addition, the maximum value of $F z$ was normalized by the user's body weight to determine the ratios of $F z$ directional force to the user's body weight.

\section{RESULTS}

Temporal changes in the vertical and horizontal forces on the foot support are presented in Fig. 3. Participants 1 and 3 showed an increase in the vertical and horizontal forces with an increase in load. However, the horizontal force did not increase even at the maximum load in participant 2, who showed a slightly different pattern compared with the other participants. The horizontal force briefly exceeded $200 \mathrm{~N}$ in participant 1 .

Table 1 summarizes the maximum and minimum values of \%Vertical, \%Vertical $\left(t_{\max }\right)$, and \%Horizontal $\left(t_{\max }\right)$ for each participant. Although a temporal change was observed in \%Vertical, the vertical force accounted for $70-80 \%$ of the total load with maximum $\%$ Vertical of $93 \%$ and minimum \%Vertical of $58 \%$. The vertical force when the maximum load was exerted, $\%$ Vertical $\left(t_{\max }\right)$, ranged from $64.7-84.1 \%$, and the horizontal force ranged from $15.9-35.3 \%$.

The maximum load on the foot support and normalized value by body weight (\%weight) for each participant are shown in Table 2. The maximum load among the three participants was $525 \mathrm{~N}$ (momentary value). As the body weight of this participant was $40 \mathrm{~kg}$, a 1.34 times higher \%weight was seen.

\section{DISCUSSION}

In the current study, the loads on the foot support were measured in three individuals with cerebral palsy. As expected, the results confirmed that the vertical force on the foot support accounted for $70-80 \%$ of the total load, whereas the horizontal force also accounted for $20-30 \%$. The horizontal force reached $35 \%$ at the maximum load in one participant. Bush et al. ${ }^{9)}$ reported that the load on a foot support was about $100 \mathrm{~N}$ when measuring loads for each support site in healthy men in a seated position. The results from all three participants in the present study were much higher than $100 \mathrm{~N}$. Moreover, that the horizontal force alone exceeded $100 \mathrm{~N}$ demonstrated that considerable loads were placed on the foot support because of spasticity. We confirmed that measuring three-dimensional loads, including the horizontal components, using a three-axis force plate should be conducted in the future in a full-scale study.

The accreditation standard for seated postural support device parts established by the Ministry of Health, Labour, and
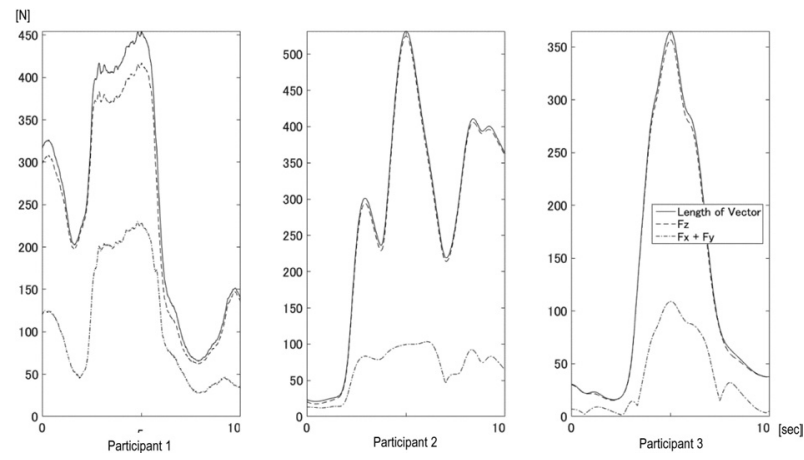

Table 1. Ratios of the vertical and horizontal components

\begin{tabular}{lccc}
\hline & Participant 1 & Participant 2 & Participant 3 \\
\hline Max. \%Vertical & 82.6 & 84.8 & 93.1 \\
Min. \%Vertical & 59.8 & 58.3 & 64.6 \\
\%Vertical $\left(t_{\max }\right)$ & 64.7 & 84.1 & 76.6 \\
\%Horizonal $\left(t_{\text {max }}\right)$ & 35.3 & 15.9 & 23.4 \\
\hline
\end{tabular}

Fig. 3. Temporal changes in the load vector, and vertical and horizontal components

\begin{tabular}{lccc}
\hline & Participant 1 & Participant 2 & Participant 3 \\
\hline Max. load (N) & 417.0 & 525.0 & 356.7 \\
\%Weight & 78.8 & 133.9 & 104.0 \\
\hline
\end{tabular}


Welfare ${ }^{4}$, which defines the mechanical strength of the foot support, requires the implementation of a downward static loading test at $500 \mathrm{~N}$ for a device for users with a body weight between 25 and $50 \mathrm{~kg}$ to ensure that there is no functional failure. A burden exceeding $500 \mathrm{~N}$ was loaded on the foot support in only one participant in this study. A load briefly exceeding the standard value is unlikely to lead directly to a breakage, because these devices are manufactured with some allowance in load bearing; however, if such a condition persists or is intermittently repeated for a long time, the likelihood of breakage increases. A long-term study in which individuals are fitted with measuring devices throughout their daily lives may be necessary in the future.

The present preliminary study included three participants; thus, the maximum loads on the foot support can be quantified but not standardized on the basis of these results alone. However, the results of this study provide important reference information for implementing a full-scale study. It is extremely important to implement a quantitative study of the actual loads on devices and report the results to establish test methods and standard values for the mechanical strength of devices in the future. This information will lead to more appropriate evaluations and product development, which will improve the everyday lives of users of wheelchairs and seated postural support devices.

In conclusion, we recorded loads caused by severe spasticity on the foot support of a wheelchair using small force plates in three participants with spastic cerebral palsy as a preliminary measurement for a more comprehensive future overload study. The results of the analysis showed that the vertical force on the foot support accounted for $70-80 \%$ of the total loads. However, the horizontal force also reached about $35 \%$ at its maximum; therefore, implementing a three-dimensional measurement using a three-axis force plate is advantageous in a full-scale future study.

\section{ACKNOWLEDGEMENT}

This work was supported by the Health and Labour Sciences Research Grant ITAKUHI (2014) and the Health and Labour Sciences Research Grant (2015).

\section{REFERENCES}

1) O’Sullivan SB, Schmitz TJ, Fulk G: Physical Rehabilitation 6th ed. In: The prescriptive wheelchair. New York: F.A. Davis, 2013 , pp 1403-1444.

2) International Organization for Standardization: Wheelchair seating-Part 3: Determination of static, impact and repetitive load strength for postural support devices. ISO 16840-3, 2014.

3) Japanese Industrial Standards Committee: Manual wheelchair. JIS T9201, 1996.

4) Ministry of Health: Labour and Welfare: accreditation standard and standard confirmation method for seated postural support device parts (second edition), 2011.

5) Young RR: Spasticity: a review. Neurology, 1994, 44: S12-S20. [Medline]

6) Aikawa T: Research on creating evaluation criteria for seated postural support devices. Report of Japanese Government Fellowship Program for the Comprehensive Research on Disability Health and Welfare, Health Labour Sciences Research Grant 2006-2008, 2009 (In Japanese).

7) Hirose H: Research on promotion of safety and appropriate distribution of seated postural support devices. Report of Japanese Government Fellowship Program for the Comprehensive Research on Disability Health and Welfare, Health Labour Sciences Research Grant 2009-2011, 2012 (In Japanese).

8) International Organization for Standardization: Wheelchairs-Part 8: Requirements and test methods for static, impact and fatigue strengths. ISO 7176-8, 2014 .

9) Bush TR, Hubbard RP: Support force measures of midsized men in seated positions. J Biomech Eng, 2007, 129: 58-65. [Medline] [CrossRef] 